\title{
FITNESS FOR SERVICE ASSESSMENT OF DEGRADED CANDU FEEDER PIPING - CANADIAN REGULATORY EXPECTATIONS
}

\author{
John C. Jin ${ }^{1}$, Raoul Awad ${ }^{2}$ and Thomas Viglasky ${ }^{3}$ \\ ${ }^{1}$ Specialist, Operational Engineering Assessment Div., Canadian Nuclear Safety Commission, (john.jin@cnsc-ccsn.gc.ca) \\ ${ }^{2}$ Director, Operational Engineering Assessment Div., Canadian Nuclear Safety Commission \\ ${ }^{3}$ Director General, Directorate of Assessment and Analysis, Canadian Nuclear Safety Commission
}

\begin{abstract}
Allowance for the continued operation of feeder piping at CANDU stations, which is experiencing active degradation mechanisms, has been based primarily on augmented inspection practices and conservative fitness for service assessments. The major degradation mechanisms identified to date are: pipe wall thinning due to Flow Accelerated Corrosion (FAC) and Intergranular Cracking due to Stress Corrosion Cracking (SCC) and potentially Low Temperature Creep Cracking (LTCC) mechanisms. Given that currently available industry codes and standards do not provide sufficient guidelines/criteria for assessing the degradation of feeder pipes, the Canadian Nuclear Safety Commission (CNSC) has asked the utilities to establish feeder pipe specific procedures to provide reasonable assurance that the risk associated with the feeder degradation is maintained at an acceptably low level. In response to this requirement, the Canadian CANDU industry has developed and continued to update a feeder fitness for service guidelines to provide evaluation procedures and industry standard acceptance criteria for assessing the structural integrity of the feeder pipes. The scope and frequency of inspections are determined based on the results of a fitness for service assessments and taking into account of the relative susceptibility of feeder pipes to each specific degradation mechanism. While industry practices for management of degraded feeder pipes have, in general, been complied with the regulatory expectations, outstanding issues still remain. Major regulatory concerns include uncertainties associated with limitations in both the inspection techniques and the mechanistic understanding of the degradation processes, which can impede inspection planning and fitness for service assessments.

This paper presents the regulator's view of the current situation with respect to degradation of feeder piping, its implications for nuclear safety and the regulatory expectations on industry's management of the critical ageing phenomena.
\end{abstract}

\section{DEGRADATION MECHANISMS AFEECTING CANDU FEEDER PIPES}

Feeder piping is an integral part of a CANDU plant's Primary Heat Transport System carrying pressurized heavy water $\left(\mathrm{D}_{2} \mathrm{O}\right)$ from the reactor fuel channel to the steam generators. The number of feeders differs from station to station, ranging from 380 inlet/outlet pairs to 480 inlet/outlet paris, depending upon the reactor design. The nominal diameter of feeder pipes across the reactor face ranges from 1.5 inches to 3.5 inches and most pipes are Schedule 80. They are manufactured from ASME SA-106 Grade B carbon steel. Among various on-going degradation mechanisms, pipe wall thinning due to flow accelerated corrosion (FAC) and intergranular cracking due to stress corrosion cracking (SCC) and potentially low temperature creep cracking (LTCC) have been identified as major degradations that may affect the operating life of feeder pipes.

\section{Flow Accelerated Corrosion (FAC) Wall Thinning}

Virtually all outlet feeder pipes at all CANDU plants are experiencing pipe wall thinning due to the FAC at a rate much higher than design allowance. The FAC wall thinning is believed to be influenced by various factors including coolant temperature and velocity and feeder geometries that contribute to flow disturbances. It is generally recognized that degradation due to FAC is relatively slow and predictable process: this allows utilities to manage its impact by predicting susceptible locations and monitoring thinning rates, as a basis for predicting the remaining service life. In the wake of the Surry-2 accident in the United States [1] and the recent Mihama event in Japan [2], considerable study has been carried out around the world, improving our understanding of the parameters affecting FAC.

To date, there have been no instances of feeder pipe failure in Canada due to wall thinning. Several feeder pipes have been replaced when their wall thicknesses fell below pre-established minimum thickness criteria. Nevertheless, regulatory staff believes that on-power failure of a thinned feeder pipe cannot be rule-out. In particular, the staff's major concern is that, in the absence of an adequate ageing management program, the ultimate failure mode of thinned feeder pipe would be sudden rupture without adequate prior warning by leakage, as has been known to occur in real-world cases.

\section{Wall thinning near welds in the Grayloc hub}


During the recent post removal examination of feeder pipes extracted from the Pickering A stations to address another FAC concern, enhanced and highly-localized thinning was discovered in the vicinity of the welds. The localized thin spots have been characterized as blunt-type flaws. While a detailed root cause investigation continues, FAC is being considered as a factor which may have exacerbated the phenomenon. Destructive examinations of other types of bends suggest that degree of susceptibility to this kind of degradation depends on the design configuration of the bends or elbows.

Prior to this discovery, it had been believed that the most susceptible location to the FAC wall thinning in the feeder pipes would be the extrados of the tight radius bends. Accordingly, ageing management, including inspection scoping and fitness for service assessment, had been focused on this region. In light of the Pickering A discovery, the situation has become more complicated. The concerns associated with this form of localized degradation relate to the possible formation of sharp flaw type features, difficulties in inspecting susceptible locations and the lack of data with which to assess degradation rate.

\section{Cracking in bends and repair welds}

Intergranular cracking is an active or at least the pre-requisite conditions exist in the tight radius bends of outlet feeders at some CANDU plants. Cracks found in these bends initiated both at the inside and outside surfaces. While we lack a complete understanding of this cracking, two possible mechanisms have been proposed by the industry: 1) Stress corrosion cracking (SCC) caused by exposure to mildly oxidizing hot coolant; and 2) Low temperature creep cracking exacerbated by hydrogen. The latter mechanism has been invoked to explain cracks initiating at the outside surface. Since the creep cracking mechanism has not been observed at temperature of outlet feeders, its proponents assert that cracking is facilitated by a flux of atomic hydrogen generated by FAC at the inside surface of affected feeders [3].

Although the root cause analysis of this cracking remains inconclusive, it is generally accepted that tensile residual stress plays a critical role in initiating and propagating service induced cracks. Accordingly, cracking is considered possible at any outlet feeder bends possessing sufficient residual tensile stresses to initiate cracking. Tight-radius, high-angle bends are considered more susceptible to cracking because of the potential for higher residual stress levels. Within a tight radius bends, the extrados region is of primary concern in terms of the margin on crack stability because of the relatively high likelihood of cracking, the axial extent of tensile residual hoop stress, the reduced fracture toughness, and the degree of thinning from fabrication and FAC. Because of station-to-station differences in the fabrication process for feeder bends and variability with regard to the application of post-bending stress-relief treatments, susceptibility to feeder cracking likewise varies across Canadian CANDU plants.

To date, thirteen confirmed cases of feeder cracking have occurred in bends and all were discovered at the Point Lepreau Generating Station (PLGS) only. Of these, two cracks went through-wall resulting in leaks. However, in both the 1996 and 2001 events, the reactor was shutdown before the cracks reached critical size. Of the remaining observed cracks , all eleven had been detected during in-service inspection and had not penetrated through-wall.

There has been one instance of leaking crack at a feeder weld. The crack, in an outlet feeder pipes at the Gentilly-2 station having a similar design to Point Lepreau. The crack was intergranular and very tight; as a result, the amount of leakage was very small. Follow-up from this incident suggested that welds repaired using full-penetration excavation and subsequent re-welding should be considered susceptible to cracking.

\section{Safety Concerns Associated with Degraded Feeder Pipes}

The potential nuclear safety implications of feeder failure are: 1) the event frequency for small LOCA under normal operating conditions may increase beyond the value assumed in the Safety Analysis and Probabilistic Risk Analysis; 2) the probability of consequential small LOCA may increase under an upset or fault condition; and 3) increased leakage from the heat transport system (HTS) resulting from feeder damage may occur as a result of upset or fault transient conditions. Industry has evaluated and managed these potential implications within the feeder fitness-for-service program. The governing principle for a nuclear safety framework for managing feeder degradation is that any increase in risk to the public arising from feeder degradation must be small. The regulatory staff is reviewing the following proposals developed by the industry in light of the governing principle: 1 ) any increase in the frequency of small LOCA, arising from rupture of a degraded feeder must be small; 2) the frequency of multi-feeder ruptures, due to feeder degradation, must remain negligible under normal and elevated stress conditions; and 3) the consequences of feeder leakage under elevated stress conditions must be limited by demonstrating crack stability and no significant increase in dose to the public.

\section{MANAGEMENT PRACTICE FOR DEGRADATIONS}

\section{Augmented Inspection Scope}

As mentioned above, the service life of feeder pipes is being limited by two active degradation mechanisms under normal operation conditions, intergranular cracking and wall thinning. At present, given the limited understanding of these degradation mechanisms and the fact that effective and reliable mitigation methods are not available, the only plausible 
options are to expand the scope of inspections and apply conservative engineering assessments with due consideration of uncertainties. The regulatory staff and industry have acknowledged that a statistically-significant number of feeder pipes should be inspected and the uncertainties associated with the inspection technology should be taken into account in assessing inspection results.

\section{Fitness-for-Service Assessment}

The utilities have developed feeder pipe fitness for service guidelines [4] which rely on two types of assessment: condition monitoring assessment (CMA) to assess the past performance and forward-looking operational assessments (OA). The objective of CMA is to demonstrate that, for feeders with identified degradation, acceptance criteria have been satisfied during the previous evaluation period. In contrast, the OA assesses the likelihood that entire population of feeders will continue to satisfy acceptance criteria over the next operating period. In applying this dual-assessment approach, the utilities' assessments need to be conservative. Otherwise, the regulatory staff will insist that any deficiencies be resolved as a pre-requisite to re-starting the plant. Even if a conservative assessment requires that feeders had to be replaced, the costs of such action would pale beside the production losses incurred if a regulator were reject a non-conservative feeder assessment.

\section{Disposition}

During an in-service inspection of feeder pipes, any detected flaw, which fails to meet specified acceptance criteria must be dispositioned to the satisfaction of the regulator [5]. Service induced cracks detected in the feeder pipe have been replaced without dispositioning for operation with detected flaws. In the case of wall thinning, Canadian industry adopts an "allowable stress" methodology to establish the minimum allowable thickness, primarily based on linear-elastic stress analysis and stress limits given by the ASME Boiler and Pressure Vessel Code, Section III [6]. Design rules in Section III, Subsection NB-3600 are utilized for this purpose with the stress indices determined by detailed finite element stress analyses. Different models have been used for the cross sectional area of the thinned feeder pipes, depending on whether or not credit is given to the thicker intrados material. If the simplified equations in NB-3600 are not fully satisfied, some elements of design by analysis methodologies given by NB-3200 are adopted including detailed elastic-plastic analysis. For fitness for service assessment of possible blunt flaws near a weld, the ASME Section III and a limit load approach are used to assess flaw stability with fatigue evaluation. The possibility of a sharp radius of the flaw increasing the stress concentration is taken into account by assessing a postulated crack.

\section{REGULATORY EXPECTATIONS}

\section{General}

Although rupture of single feeder pipe falls within the envelope of design basis accidents considered in Safety Analyses for Canadian CANDU plants, the regulatory staff remains concerned about consequential effects, such as the potential for damage to other core components and the release of radioactivity to the public. Key factors contributing to this concern are current limitations in both our understanding of feeder pipe degradation mechanisms and in-service inspection capability. It is the regulator's view that reliable assessment of fitness for service of flawed components requires the integration of different aspects from several different disciplines; for example: a mechanistic understanding of degradation, material behavior, principles of engineering structural evaluation, NDE technology and so on. The limited knowledge regarding the causes of the degradation may lead to susceptible areas that are not inspected. Accordingly, regulatory staff has insisted that inspection planning and structural integrity assessments should take into account of these limitations in a conservative way. In practical terms, this means that regulatory staff allows a utility to continue operating degraded feeder pipes only when they provide a conservative engineering evaluation of the observed degradation, and commit to an expanded inspection scope to identify other feeders with similar or potentially more severe degradation.

Considering the large amount of feeder pipes in a CANDU station, it may be impractical to completely inspect each pipe during every inspection outage. An important reason is the CNSC's ALARA (As Low As Reasonably Achievable) principal. Since feeders are often located in high-radiation environments, the regulatory staff asks that licensees carefully weigh the benefits of inspecting a given feeder in terms of reducing the risk posed by unrecognized degradation against the dose penalty paid by inspectors. As a result, there is a motivation for utilities to develop strategies to focus inspection resources on those feeders ranked as having higher susceptibility to each degradation mechanism. Canadian regulatory staff has recently considered a probabilistic assessment as an aide in assessing the risk increment associated with specific type of degradation and the effect of inspection scope and frequency in reducing the risk.

\section{Service Induced Cracks}

If there is any doubt regarding the manner that a detected crack like flaw initiated and propagated under the plant's operating conditions, it shall not be evaluated for a disposition for continued operation with the detected flaws. For the 
plants where feeder pipe cracking is credible, it is expected that there must be: a) a sufficient level of mechanistic understanding, b) reliable data on flaw growth rate models supported by relevant tests, c) a reliable critical crack size determination supported by a sufficient number of proof tests and d) quantified inspection capability in terms of detection limit and sizing uncertainties. Probabilistic evaluation may be required to show that the inspection scope and frequency is adequate in reducing the risk increment associated with credible degradation mechanism to acceptable level. The issue of the detection limit and probability of detection of the ISI shall, for example, be addressed by evaluating the structural integrity of feeder pipe with postulated flaws. In that case, the size, shape and location of the postulated flaws should be included in a conservative manner.

It has been the regulatory position that management of degraded feeder pipes shall be conducted in such a way as to prevent leakage no matter how small the leak may be. The basic philosophy of licensing has been to detect flaws by inservice inspection so that the leakage of coolant does not occur. Consideration of the consequence of leakage deviates from the pressure boundary design concept which contends that the pressure boundary should be maintained. However, in very limited cases, where there can be no assurance that a crack does not exist, it is the regulatory view that the management of a degradation mechanism could be based on the consequences of a leak, but only when there is concrete evidence demonstrating final failure mode would be leak before break (LBB). In this case, the concept of the LBB shall be applicable for a specified operating period, not as a principal long term method for managing service induced degradation. The Canadian regulatory perspective on the application of the concept of LBB for the justification of continued operation of degraded components is presented in another paper [7].

\section{Blunt Flaw}

With respect to the assessment of possible blunt flaws, the major concern is that it may not be detected by the existing NDE tools, not to mention their insufficient sensitivity to characterize flaw and to confirm that no micro cracking is present in the flaw. As this type of flaw was discovered only recently in an unexpected area near the Grayloc hub weld, there is insufficient data to assess the degradation rate. A structural integrity evaluation shall be performed with the consideration of a high local stress concentration which may result in crack initiation. Regulatory expectations are: 1) mechanistic understanding should be improved to permit the prediction of susceptible locations or the susceptibility of different configurations of weld geometry, 2) inspection tools should be improved to enable detailed characterization of the flaw, and 3) if there is accessibility concern, conservative flaws should be postulated in engineering evaluations for the crack stability and fatigue initiation.

\section{Wall thinning}

Although it is arguable that degradation due to FAC is predictable and manageable, there are still concerns caused by uncertainties involved in the inspection and evaluation methodologies. Considering the complexity of the mechanistic process of FAC involving fluid and structure interaction, chemical reaction, erosion processes, and so on, it cannot be assumed that methods developed to predict susceptible locations and to assess the rate of metal loss are infallible and, therefore, conservatism should not be compromised during inspection planning activities. Obtaining accurate thickness measurements using ultrasonic testing (UT) is known to be highly dependent on the specimen geometry and surface conditions. It is also difficult to ensure that the region of wall thinning has relatively smooth contours without notches that may act as stress concentrations. Accordingly, it may be prudent to assess the impact of a crack in the FAC wall loss region because of possibility of micro cracks at the region and the inability to reliably detect such features. Uncertainties in the inspection require conservatism in the flaw model used for engineering evaluation.

It is generally considered conservative to apply an allowable stress methodology in accordance with the ASME Section III. However, there are still a number of sources of uncertainty in the process of engineering evaluation for structural integrity of degraded pipes, which could erode safety margins. The sources of uncertainties may exist in:

i) Flaw modeling: In-service inspection techniques may not be sufficiently sensitive to characterize detected flaws, introducing the possibility of un-conservative modeling results.

ii) Load calculation and combination, particularly for dynamic excitation: Feeder pipes are coupled to each other by spacers and also supported by various types of restraints, resulting in very complex dynamic behavior. Calculating seismic responses of this complex piping system may lead to un-conservative results. It can be difficult to quantify the effects of non-design basis loading, such as vibration, on the structural behavior.

iii) Residual stress: Magnitude of residual stress remaining in components and its effect on the component integrity is difficult to quantify.

iv) Degradation of material properties: It is generally known and also observed in many actual cases that material properties may deteriorate due to operating conditions. Dynamic strain ageing effects on fracture toughness also have 
been identified by proof testing particularly for feeder pipe material, carbon steel SA-106, Gr. B. Variations in material properties in the heat affected zone might be overlooked in the assessment of flaw near welds.

v) Stress classification: Classification of stresses in the flawed pipe into local/general, primary/secondary may not be straightforward. Classification into local stress should be supported by inspection results. Effect of local overstrain shall be taken into account in feeder pipe structural integrity assessment.

Therefore, it is the regulator's expectation that parameters and methodologies used in the engineering evaluations for demonstrating fitness for service shall be always performed in a conservative manner to consider many different sources of uncertainties.

\section{CONCLUSIONS}

With respect to the management of feeder pipe degradation mechanisms, the major regulatory concerns are the limitations in the mechanistic understanding of the processes involved and the uncertainties in the inspection capabilities and engineering assessments. Listed in this paper are the possible sources of uncertainties addressed from a regulator's point view. It is the regulatory position in accepting the industry's request for continued operation of degraded feeder pipes that a sufficient number of feeders must be inspected to establish a representative and reliable account of the level of degradation and that the uncertainties in the inspection techniques and engineering evaluations shall be taken into account by using conservative engineering assessment parameters and methodologies.

\section{REFERENCES}

[1] U. S. Nuclear Regulatory Commission, NUREG-1344, "Erosion/Corrosion-Induce Pipe Wall Thinning in U.S. Nuclear Power Plants”, April 1989

[2] U. S. Nuclear Regulatory Commission, Information Notice 2006-08, “Secondary Piping Rupture at the Mihama Power Station in Japan”, March 16, 2006

[3] T. Gendron, J. Slade and G. White, "Pinpointing Cracks - Why a Probabilistic Approach to Managing Coolant Pipe Cracking Was Needed at Point Lepreau”, Nuclear Engineering International, January 2007

[4] CANDU Owners Group Inc., Fitness for Service Team of COG Feeder Integrity Joint Program, "Fitness-for-Service Guidelines for Feeders in CANDU Reactors”, July 2006

[5] CAN/CSA N285.4, "Periodic Inspection of CANDU Nuclear Power Plant Components,” Canadian Standards Association, 1994

[6] ASME Boiler and Pressure Vessel Code, Section III

[7] J. Jin, A. Blahoianu and T. Viglasky, “Canadian Regulatory Perspective on LBB Application for CNADU Piping”, August 2007, Structural Mechanics in Reactor Technology (SMiRT), Toronto, Canada 